\section{Archeologia della produzione architettonica. Le tecniche costruttive}

\author{
TIZIANO MANNONI
}

\begin{abstract}
Riasunto
La maggior parte delle classificazioni dei modi di costruire murari si basa sugli aspetti formali delle superfici visibili, con certe utilità nel distinguere le unità stratigrafiche murarie e negli aspetti estetici delle superfici stesse. Dal momento però che i muri fanno parte delle strutture portanti più importanti, non si può parlare di tecniche murarie senza cercare di capire come i vari tipi classificati si comportino realmente dal punto di vista statico. Si cerca perciò di verificare e in qualche modo di capire l'intero volume di ogni tecnica muraria, e se vi siano della regole generali o particolari che siano in qualche modo deducibili anche dalle superfici visibili. Si propone infine una classificazione delle «famiglie» di tecniche murarie più note sulla base dei loro sistemi costruttivi e funzionali, oltre che formali.
\end{abstract}

Parole chiave: Archeologia dell'Architettura, Scienza delle Costruzioni, Tecniche Murarie

\begin{abstract}
In the field of the building techniques used in the construction of the walls, the majority of the classifications consists of the formal aspects of visible surfaces. These classifications use some attentions distinguishing stratigraphic units and in the field of the aesthetic aspects of surfaces. As the walls take part of the most important load-bearing structure, isn't possible to speak of the techniques used in the construction of the walls without trying to understand how the different types of wall effectively operate from a static point of view. So it's necessary to check and to understand the whole volume of each technique used in the construction of the walls, and if there are general or particular rules deductibles from the visible surfaces. In this paper we suggest, also, an classification of the «families» of the most recognized techniques used in the construction of the walls, on the basis of their constructive, functional and formal systems.
\end{abstract}

Key words: Building archaeology, Building science, techniques used in the construction of the walls
Premesse. Se archeografia vuol dire «descrizione oggettiva dei reperti», e archeometria «dati quantitativi, e non, forniti dai reperti con strumenti delle scienze naturali», archeologia significa «discussione critica di tutte le informazioni oggettive presenti negli oggetti e negli ambienti del passato». La descrizione oggettiva porta automaticamente alle classificazione dei reperti: strumenti induttivi basati sulle osservazioni, rappresentazioni e misure, che devono, cioè, essere continuamente verificati, ed eventualmente aggiornati, mano a mano che nuovi reperti vengono studiati, ma essenziali per le discussioni critiche e per non cadere nel vago o nel soggettivo. Anche i dati forniti dalle scienze naturali possono venire classificati (per esempio muri di granito o muri di calcare), oppure possono entrare nelle classificazioni archeografiche.

La discussione archeologica ha lo scopo principale di interpretare storicamente tutti i dati raccolti, ma non è possibile affrontare razionalmente in una stessa discussione tutti i modi nei quali può essere osservata una società del passato, senza contare che molti modi ci sono ancora ignoti: questo non è possibile neppure osservando una società attuale. Essendo nata l'archeologia nell'ambito della storia dell'arte, come strumento analitico, è normale che l'aspetto prevalente sia stato per molto tempo, e sia ancora, quello formale nel senso estetico, esteso dai valori unitari dell'opera architettonica alle caratteristiche stilistiche degli elementi architettonici più rappresentativi, e quasi simbolici, ma anche agli elementi essenzialmente strutturali, come i muri.

Non vi è dubbio che, specialmente quando un edificio doveva rappresentare per qualche motivo la perfezione estetica, anche gli elementi portanti venissero eseguiti con forme ed effetti superficiali dei materiali che, senza diminuire le qualità strutturali, le superassero suscitando associazioni con espressioni di armonia, perfezione ed «eternità», in accordo e rinforzando la bellezza architettonica globale. Questo comportava ovviamente, come dice chiaramente Vitruvio, e come ripetono per diciotto secoli gli autori dei trattati di architettura, che il progettista, con l'aiuto o assieme al costruttore, conoscesse bene le caratteristiche dei materiali non solo dal punto di vista cromatico, ma anche della lavorabilità, della resistenza meccanica e della durata agli agenti di degrado; non solo, ma che conoscesse bene anche le tecniche migliori per la loro estrazione, lavorazione e messa in opera ai fini della resistenza, della durata, ma anche degli aspetti estetici e funzionali dell'opera.

Le funzioni pratiche degli edifici sono sempre state assai differenti e ciascuna di esse richiedeva la creazione di ambienti con caratteristiche spaziali, climatiche, di illuminazione e di resistenza all'usura diverse in rapporto all'ambiente esterno. Come si vede l'ideazione architettonica doveva soddisfare in modo unitario esigenze di natura assai differente, ma tutte 
ugualmente importanti: era una situazione diversa da quelle della pittura e della scultura; era più vicina a quella della produzione di oggetti mobili belli, ma funzionali; però questi ultimi raramente potevano raggiungere come le costruzioni valori estetici molto forti nel caratterizzare il paesaggio umano, e i rapporti fra materiali, strutture, funzioni e bellezza in architettura sono sempre stati assai più complessi.

$\mathrm{Si}$ è visto che le descrizioni archeografiche e i dati archeometrici non sono sufficienti nel costruito, più che in altre produzioni, a fornire le informazioni necessarie per una corretta discussione archeologica. Sono normali le domande di questo genere: la scelta di quel materiale era opportuna? La migliore che si potesse fare? Esisteva già da tempo o era innovativa in quel territorio? Quale conoscenza richiedeva? Lo stesso vale per quanto riguarda una tecnica costruttiva o per una soluzione strutturale. In architettura, cioè, è molto evidente che tutti i rapporti delle caratteristiche naturali dell'ambiente e delle sue risorse con le funzioni, la durata e l'estetica delle costruzioni è sempre dipesa da un insieme di «saper fare» trasmessi in ogni società: la cultura materiale fa da ponte logico tra i dati archeografici, quelli delle fonti scritte ed orali (etnoarcheologia) e quelli archeometrici, fornendo anche le basi per l'archeologia sperimentale. I suoi principi sono stabiliti dal come funziona, e ha sempre funzionato, la scoperta empirica di un saper fare (tentativi ed eliminazione degli errori) e la sua trasmissione (apprendimento delle «regole dell'arte» direttamente da un maestro).

I rapporti tra il «saper fare» e i dati scientifici sono validi perché la ricerca empirica è razionale e, pur limitandosi agli effetti e non avendo come scopo, a differenza della scienza, la scoperta delle cause dei fenomeni naturali, arriva in tempi lunghi a scegliere le tecniche e i materiali più adatti. Oggi possiamo in più sapere perché tali scelte siano le migliori, fatto che modifica radicalmente il modo ed i tempi di avanzamento della conoscenza e delle sue applicazioni, ma ciò non toglie che la corrispondenza fra i risultati ottenuti con i due metodi esista, come le ricerche archeometriche vanno continuamente dimostrando. Anzi, vi sono casi in cui si cercano scientificamente le cause di certi ottimi comportamenti dei materiali antichi di cui si è interrotta la trasmissione del sapere empirico, per recuperare qualcosa di ancora utile, come è il caso delle calci magnesiache (Metodo I.S.CU.M.). Data la complessità fisico-chimica di questi materiali, forse la ricerca scientifica non se ne sarebbe curata, se non vi fossero prove molto estese della loro qualità nel costruito storico.

\section{FUNZIONI E STRUTTURE}

A differenza della maggior parte dei manufatti mobili, se si escludono i vari generi di recipienti, le principali funzioni delle opere architettoniche sono esercitate dai vuoti più o meno grandi che esse determinano impedendo l'ingresso in essi degli agenti ambientali e creando, perciò, particolari ambienti artificiali. L'insieme delle strutture serve quindi, in senso funzionale, a determinare nel migliore dei modi questi vuoti, ma la loro funzione primaria, a questo scopo, è quella di stabilire e mantenere nel tempo un equilibrio statico. Da quando i primi paleolitici hanno imparato a incrociare e legare dei pali di legno per creare una tenda, questo abbinamento struttura-funzione si è molto evoluto, ma non è sostanzialmente cambiato.

Quando dalla tenda si è passati alla capanna si sono praticamente aggiunte sotto una struttura coprente delle strutture verticali che sorreggono la prima, ma che creano anche spazi utili più grandi. Finché però le strutture verticali erano costituite da montanti di legno, l'eventuale impiego di pietre o mattoni nei muri non era portante, ma serviva solo a tamponare i vuoti lasciati dai pali o dai telai di legno. Nei territori dove però il legno era meno disponibile, esso è stato presto limitato alle coperture: strutture per le quali il suo peso specifico inferiore e, soprattutto, la sua ottima resistenza alla flessione, a differenza di quella delle pietre, lo rendeva più difficilmente sostituibile. In questo caso si è imparato a sovrapporre delle pietre di dimensioni manovrabili, dato il loro alto peso specifico, fino a creare i primi muri portanti.

Basta provare a fare un muro a secco per capire che non si tratta di una operazione semplice come può sembrare a prima vista. La costruzione del muro a secco è, in cambio, una ottima palestra sperimentale per capire se esistano delle regole empiriche e quali esse siano. L'equilibrio statico di una struttura verticale, se si considera come un corpo unico ed omogeneo, non è difficile, nel senso che è sufficiente che essa sia realmente a piombo (senza cioè inclinazioni o aggetti che spostino troppo il suo baricentro), che il suo spessore in basso sia sufficiente per reggere il peso soprastante, e che il suolo in cui è fondata sia omogeneo, orizzontale e anch'esso resista bene al peso. Questo va bene per le strutture monolitiche che richiedono però una organizzazione costruttiva gigantesca, se si vogliono raggiungere opere di un certo volume. Per ottenere lo stesso comportamento statico con una struttura verticale non monolitica, ma costruita giustapponendo elementi litici più piccoli, bisogna che esista anche un buon equilibrio fra tutti gli elementi che la compongono.

Questo equilibrio si può imparare nel modo migliore costruendo senza leganti, perché in questo modo i pesi si scaricano direttamente da pietra a pietra (materiale in genere più resistente dei leganti), ed è più facile vedere subito quanto siano estesi e stabili i punti di contatto tra elemento 
ed elemento. Esistono muri storici con un legante di terra argillosa: in quelli ben costruiti la terra si limita a sigillare i vuoti con scopi di isolamento termico e dell'umidità, e non statici. Anche con i leganti a base di calce non si deve pensare che il muro stia assieme ad opera della malta, come se fosse un calcestruzzo (bassa aderenza e resistenza alla trazione della malta); quando i contatti orizzontali fra gli elementi sono stabiliti da un cuscino di malta, la resistenza globale alla compressione sarà quella della malta stessa, sempre inferiore a quella della pietra (basta aumentare in proporzione lo spessore della struttura).

\section{TIPOLOGIA DEI MURI}

Le classificazioni dei muri che non tengono conto della loro lunga storia funzionale sono basate sulle osservazioni oggettive delle forme delle loro superfici esterne, e possono certamente servire per confronti tipologici e, quando in un territorio certi tipi siano legati a periodi circoscritti, possono essere anche impiegate come indicatori cronologici di genere indutttivo.

Chiunque nota che esistono tipi assai diversi di muri di pietra, anche fra quelli che a prima vista possono sembrare simili tra loro. Non sono tanto il colore e la grana della pietra che colpiscono quanto la lavorazione e la disposizione dei singoli elementi. Se ci si lascia dominare nel giudizio dal piacere estetico che le opere murarie possono infondere nell'osservatore, a volte anche in modo subconscio, la tendenza prevalente è quella che considera belli i muri «privi di difetti esecutivi»: quanto più la materia non appare nella sua forma grezza e naturale, quanto più la disposizione degli elementi è geometricamente regolare, tanto più l'opera sembra armoniosa e tendente al perfetto e al sublime. In quei muri nei quali la lavorazione non è geometricamente perfetta, vi possono essere modi di costruire che, se presenti, ne richiamano l'effetto, come il mantenere dei corsi, ciascuno con altezza costante, o anche il solo regolarizzare il piano ogni tre o quattro corsi; dopo queste minime geometrie si ha una specie di incomprensione formale e si pensa che sarebbe meglio coprire il muro con un rivestimento.

Un reticolo regolare di rettangoli non ha tuttavia simmetrie e rapporti tali da costituire una speciale bellezza formale; è probabile quindi che si tratti in parte di un piacere rinforzato da associazioni suscitate dalla quantità e dalla precisione del lavoro, ma anche dalla resistenza e durata della pietra come materiale, dalla disposizione perfettamente orizzontale e verticale dei giunti e dal perfetto incastro degli elementi d'angolo; il tutto legato alla forma architettonica di cui il muro fa parte.

Non a caso, infatti, tutti pensano che i muri belli siano anche i più resistenti, quasi eterni. L'archeologia dei muri ci insegna invece che fra le opere che hanno meglio sfidato il tempo ve ne sono di appartenenti a tutti i tipi. Lo stesso Vitruvio parla di difetti che possono esistere in certe opere regolari e della buona durata di opere irregolari. Lapproccio puramente tipologico è quindi inadatto quando si voglia studiare la storia dei modi di costruire e l'organizzazione dei cantieri e delle loro forniture. Senza contare che una classificazione che anteponga agli aspetti formali quelli tecnologici potrebbe, come è già avvenuto in altri settori dei reperti archeologici, migliorare e rendere più agile e più vicina alla realtà le tipologie dei muri.

\section{TECNICHE MURARIE}

Per tecnica muraria, prima di tutto, non si può intendere soltanto ciò che si osserva sulle superfici: le proprietà meccaniche di un muro investono tutto il suo spessore, e di conseguenza, come si vedrà, anche le sue regole costruttive. Neppure il concetto di paramento coincide del tutto con la forma visibile sulla superficie: tecnicamente un paramento esiste quando lo strato esterno dell'opera muraria viene eseguito in modo diverso dall'interno del muro, e si intende perciò l'intero apparecchio che costituisce tale strato: per esempio, una superficie costituita da conci perfettamente squadrati spesso corrisponde ad una vera opera quadrata, con conci portanti; altre volte si ha invece a che fare con un rivestimento che regge solo su se stesso. In questo caso la differenza è rilevabile anche in superficie, se si tiene conto degli spessori dei conci visibile nei cantoni, ma questo non influisce sulla percezione formale globale, tanto è vero che spesso chi osserva dal punto di vista storico artistico i muri non si accorge della differenza.

La maggior parte dei muri non è studiabile in modo tridimensionale, tranne quel poco che si può dedurre dalle osservazioni e dalle misure dei cantonali, e solo in qualche caso dove si può rilevare con sicurezza la presenza di conci passanti da una superficie esterna all'altra. Il rilievo e l'analisi completa di un muro si può fare solo in situazione di rudere o durante i restauri di un'opera che era stata in passato tagliata. Dopo un certo numero di casi, però, quando si è cioè verificato che ad un certo tipo di paramento o di forma delle superfici, corrisponde sempre una tecnica globale ben precisa, è possibile collegare i tipi alle tecniche. Trattandosi di un metodo induttivo, bisogna tuttavia verificare se l'abbinamento funziona in tutti i casi in cui ciò è possibile, onde ampliare o abolire le corrispondenze, ove fosse necessario.

Lo studio tridimensionale è quello che ha anche permesso di constatare che sono sempre esistite alcune regole per la messa in opera degli elementi litici che costituiscono un muro; regole valide ed uguali per qualsiasi tipo di tecni- 

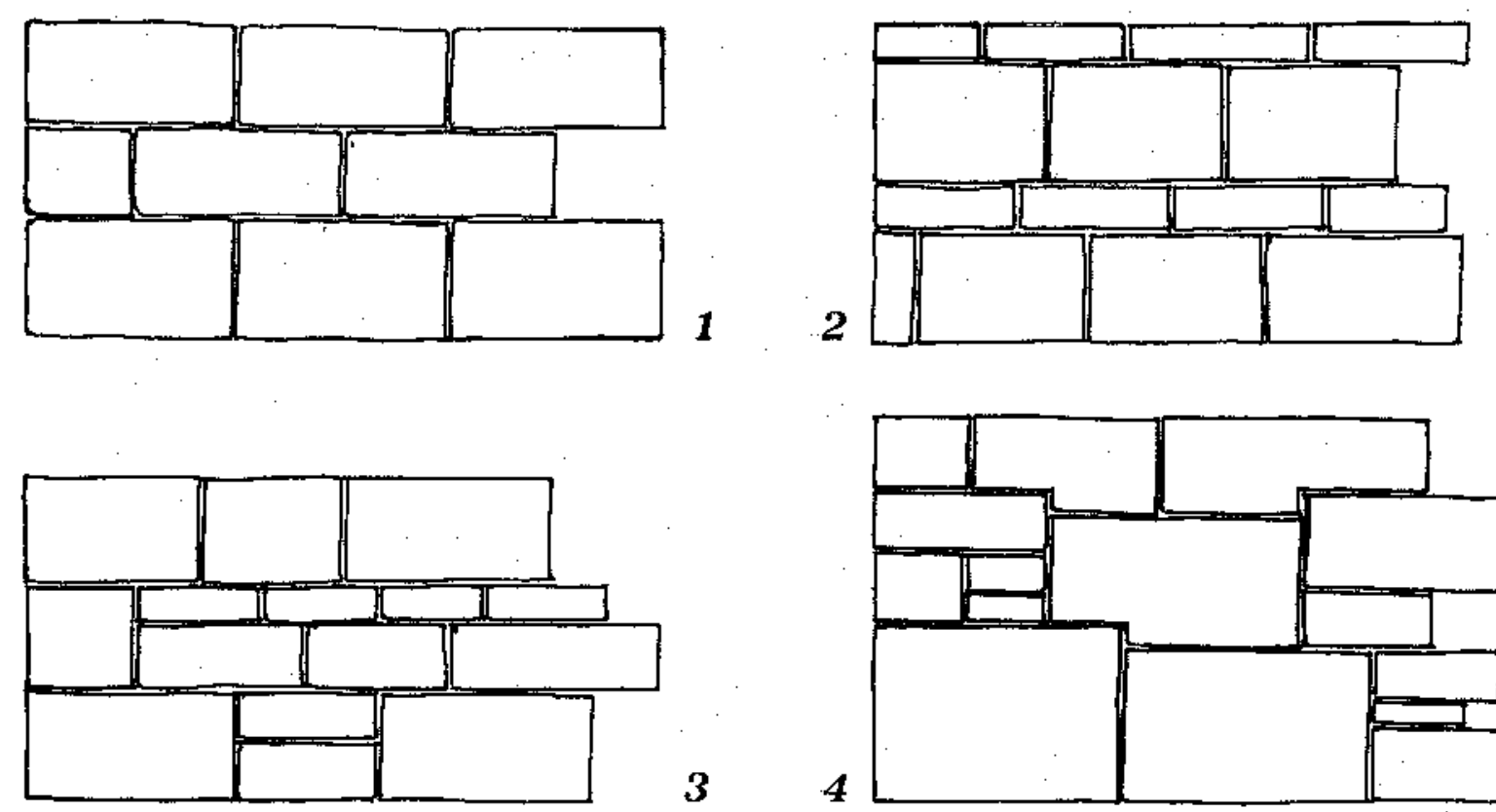

3
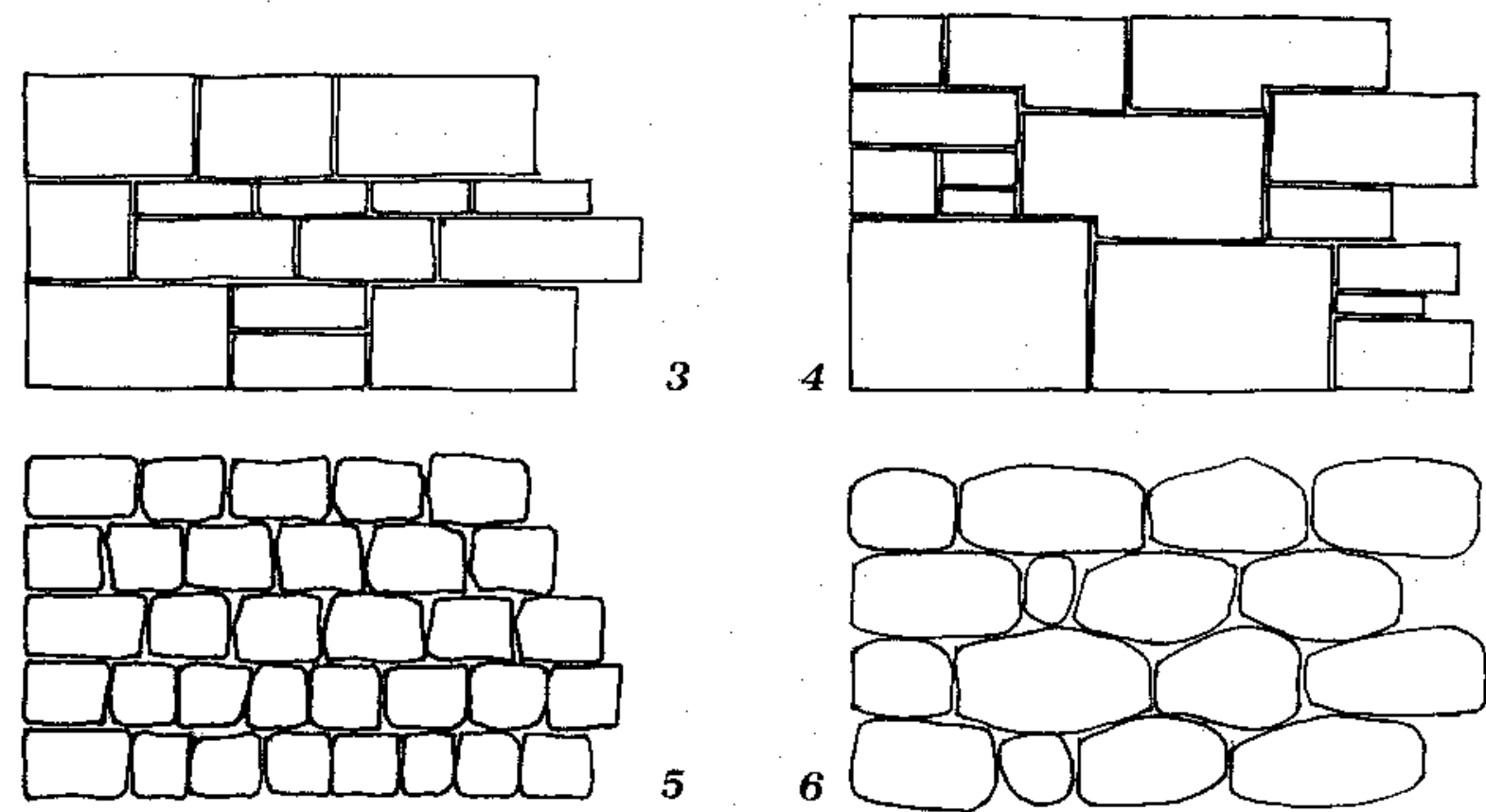

5
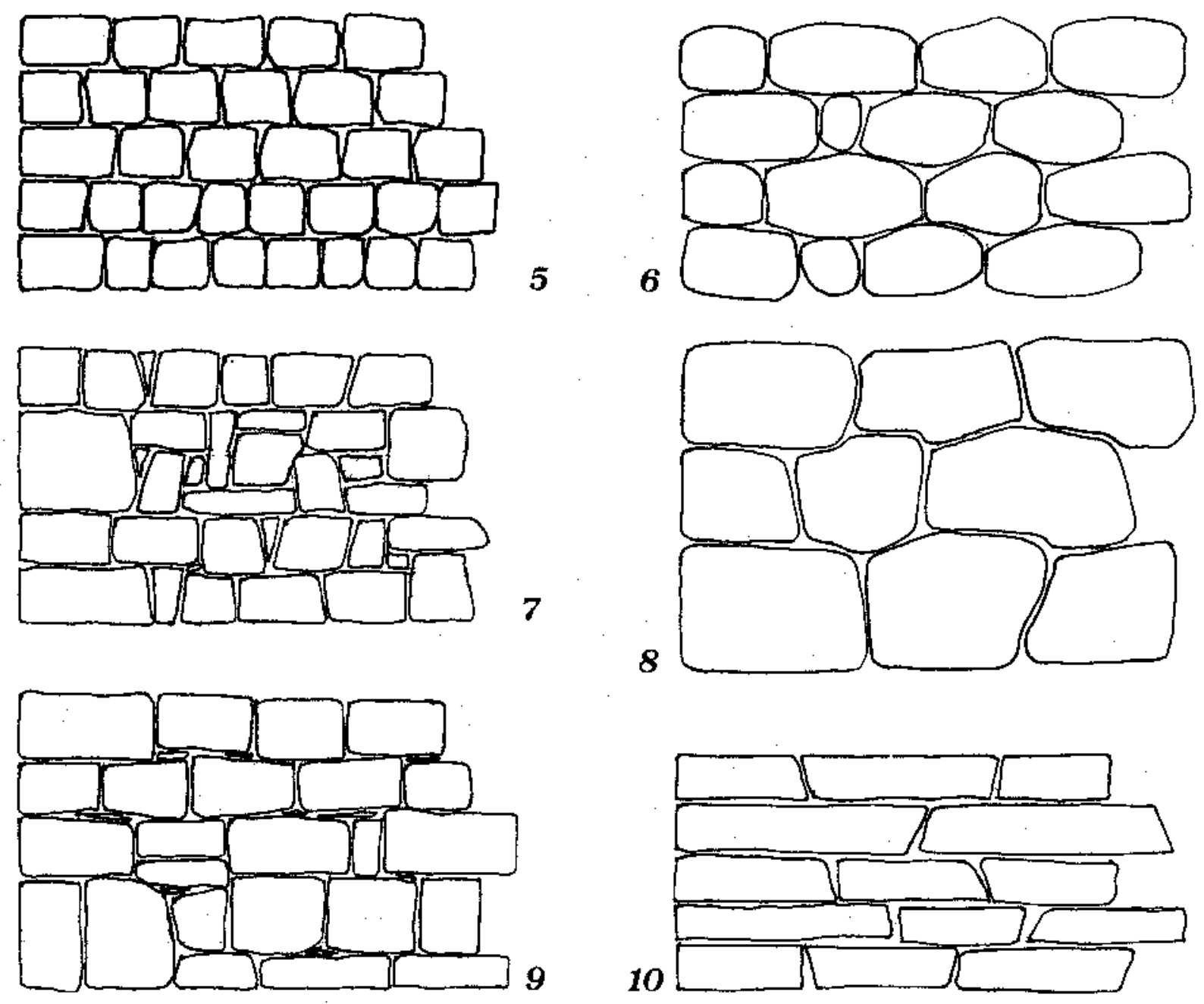

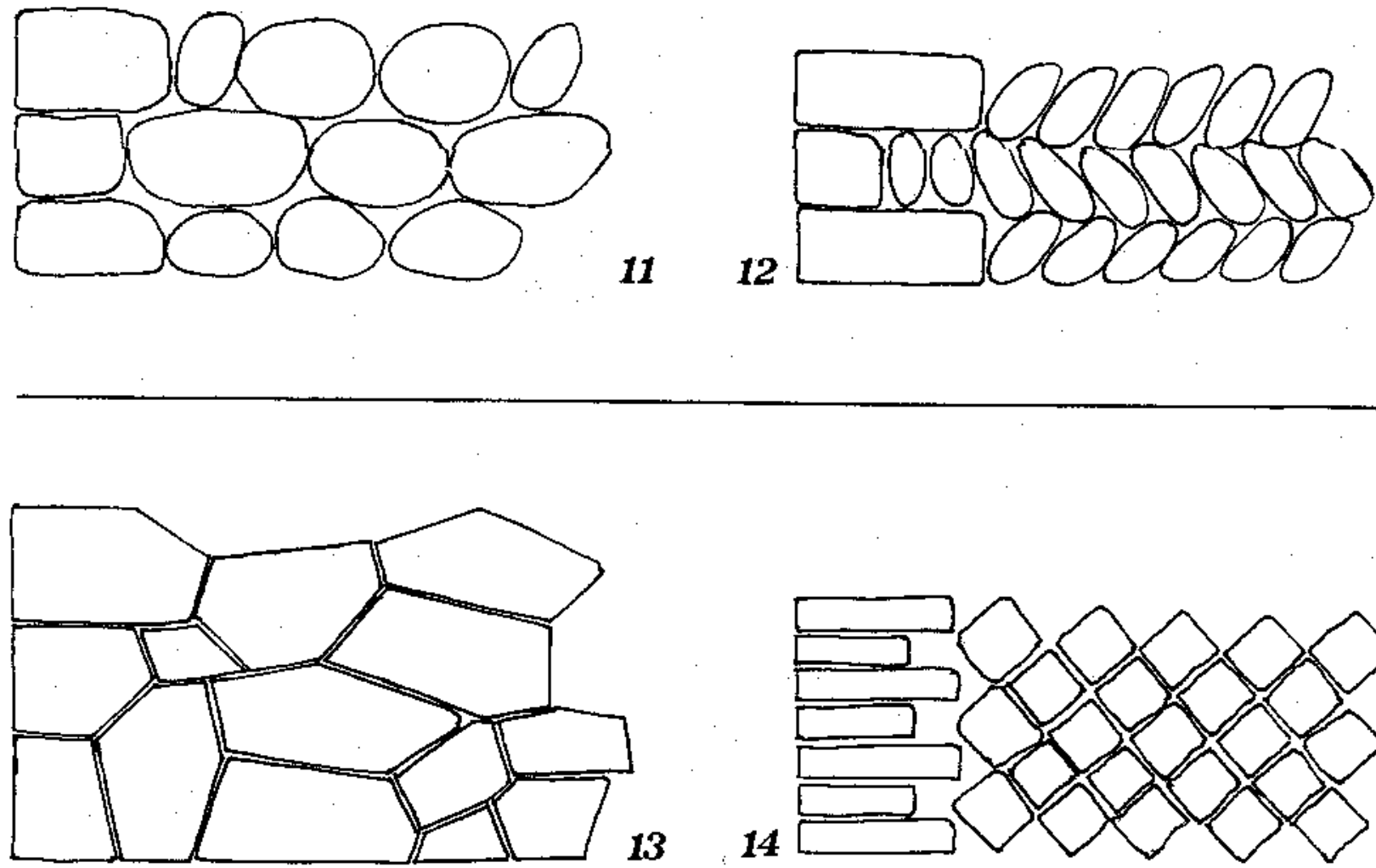

13

14
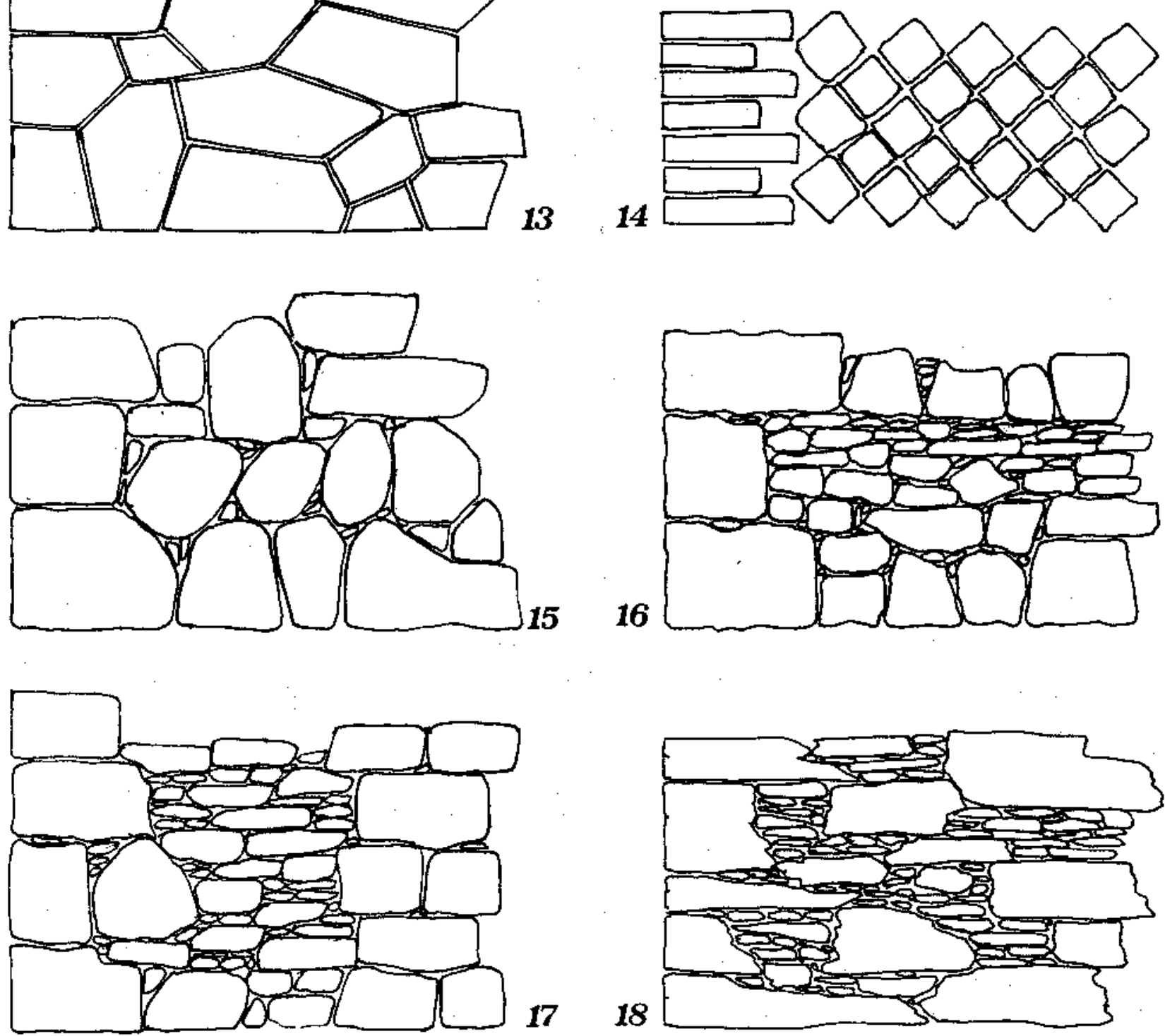

17

18

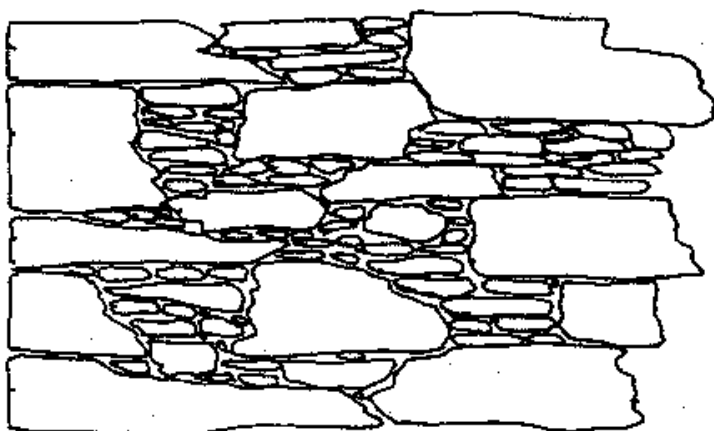

I principali tipi di paramento murario con rispettive angolate. $A$ corsi (B1): 1) regolari di conci squadrati; 2) alternati di conci piatti; 3) sdoppiati di conci differenti; 4) a scalini di conci differenti; 5) regolari di bozze uguali; 6) budinati di ciottoli sbozzati; 7) con ripiani ogni due o tre file di bozze differenti; 8) budinati di grandi bozze poligonali; 9) sdoppiati di bozze con zeppe; 10) regolari di strati naturali spaccati; 11) regolari di ciottoli grandi; 12) regolari di ciottoli piccoli a «spina-pesce». Senza corsi (B2): 13) poligonale a conci spiattati; 14) reticolato a bozze uguali; 15) poligonali a bozze differenti con zeppe; 16) complesso con bozze e scarti «a giornate» (dai pezzi più grandi a quelli più piccoli); 17) complesso con bozze e scarti «a pilastri»; 18) complesso con pietre naturali e scarti disposti «ad alveare». 
ca impiegata e che trovano accordi e spiegazioni per un buon equilibrio statico all'interno del muro stesso. È pure evidente che altre regole si aggiungono a quelle generali quando si passi dalle tecniche più disordinate a quelle più ordinate, volendo mantenere per ora i termini desunti dalla tipologia muraria basata sulle prime osservazioni delle superfici esterne.

Già in questo primo approccio la cultura materiale mette in luce, prima di tutto, che nella maggior parte delle tecniche murarie è semprestato il muratore vero e proprio che applicava il saper fare acquisito dai suoi maestri: dava istruzioni sull'approvvigionamento delle pietre e sulle loro eventuali preparazioni per l'uso; preparazioni che spesso effettuava o completava egli stesso mano a mano che murava. Differenti erano i casi delle tecniche con pietre spaccate e sbozzate con misure standardizzate (litostrati costanti, tuffelli, ecc.), quando cioè il muratore si trovava ad operare con elementi tutti uguali come se fossero dei laterizi; ma diversi erano anche i casi dei vari tipi di opera quadrata, nei quali il muratore si limitava a mettere in opera ciò che progettava ed eseguiva lo scalpellino: i paramenti del muro cioè venivano prefabbricati in tutti i dettagli a terra.

Un altro procedimento necessario a questo punto è quello che separa le regole sulla lavorazione precisa dei singoli elementi litici, che erano proprie dello scalpellino, dalle regole del muratore, ovvero della messa in opera, o collocazione spaziale in tre dimensioni degli elementi costruttivi di qualsiasi forma, anche quando si trattava di corsi regolari, regole di cui anche lo scalpellino doveva tenere conto: non tutti i conci perfettamente squadrati sono posti in corsi regolari e, soprattutto, non tutti i corsi regolari sono fatti di conci perfettamente squadrati.

Traducendo la cultura materiale dei muri in regole generali scritte, valide per qualsiasi tecnica, esse potrebbero essere formulate nel modo seguente.

I. Ogni elemento litico deve appoggiare su almeno due di quelli sottostanti, se si vuole «legare l'opera» sia nei paramenti, sia all'interno e sia se i paramenti non esistono, facendo in modo che non si formino pilastri separati nel senso della lunghezza del muro (migliore distribuzione dei carichi concentrati).

II. Fare in modo che ogni elemento appoggi in più punti possibili su quelli sottostanti, sia sfruttando l'adattamento delle superfici naturali o il perfetto spianamento dei conci, sia con l'impiego di zeppe ottenute con scaglie di pietra sana, di giusta misura e infisse a martello fino alla costipazione, evitando sollevamenti: non attribuire alla malta questo compito, ma soltanto quello di saldarne i rimanenti spazi (aumento della omogeneità dell'opera e della sua resistenza alla compressione)
III. I piani di posa visti sulle superfici esterne nel senso longitudinale devono essere orizzontali da un cantone all'altro. Quando ciò non sia possibile a causa degli elementi geometricamente irregolari impiegati, le zone dei cantoni devono comunque rispettare questa regola (evitare tensioni interne al muro nel senso della sua lunghezza).

IV. I piani di posa visti nella sezione trasversale devono essere sempre orizzontali. Per nessuna tecnica è ammessa un'eccezione (evitare tensioni interne al muro nel senso perpendicolare alle superfici esterne).

V. La maggiore lunghezza degli elementi litici va spesso collocata in modo perpendicolare alla superficie esterna nei muri privi di paramenti; in quelli con paramenti un concio ogni tanto deve collegare le due opposte superfici esterne, onde non si formino pilastri separati nel senso della larghezza (migliori distribuzioni dei carichi concentrati).

VI. Una regola infine riguarda le tecniche che prevedono piani di posa non orizzontali nel senso della lunghezza del muro. Oltre a quanto già affermato nella regola III per quanto riguarda le zone dei cantoni, ogni altro elemento litico deve appoggiare sui due sottostanti con due inclinazioni di senso opposto, in modo che le tensioni che si determinano nel senso longitudinale del muro stesso si compensino il più possibile tra loro (aumento della omogeneità dell'opera).

\section{INTERPRETAZIONI}

Tutte le regole sembra che tendano in un modo o in un altro a far sì che il muro, con qualsiasi tecnica sia stato realizzato, sia il più possibile omogeneo dal punto di vista meccanico in tutte le sue parti, e si comporti perciò come una struttura uniforme, senza perdere tuttavia quella elasticità che gli deriva dall'essere composto da molti elementi assemblati e che si è dimostrata molto utile in rapporto, per esempio, ai terremoti.

Le tecniche esteticamente poco piacevoli non sono perciò anche poco resistenti, e questo spiega la loro reale sopravvivenza alle prove del tempo al pari delle tecniche belle. Non si può parlare per conseguenza neppure di tecniche ordinate, o regolari, e disordinate o irregolari, perché l'apparente disordine formale non corrisponde ad una mancanza di regole strutturali. Come al solito, quando l'ordine non è di immediata evidenza, non corrisponde cioè a formule e geometrie semplici, viene catalogato come disordine o casualità: è più esatto allora parlare di tecniche complesse e tecniche semplici.

Che la tecnica dell'opera quadrata sia concettualmente semplice non vi è dubbio: la soluzione idealmente più semplice è proprio quella di costruire con cubi tutti uguali, dello spessore stesso del muro, sfalsandoli di mezzo lato ad 
ogni corso. Questa sarebbe sempre stata però anche l'operazione più costosa perché avrebbe richiesto una fornitura e lavorazione di blocchi sempre sufficientemente grandi e avrebbe creato una notevole quantità di scarti. Tecnica semplice non significava quindi di più breve esecuzione e perciò meno costosa. Anzi, al contrario, le più veloci e meno costose sono sempre state proprio le tecniche complesse: quando una scuola regionale di muratori aveva messo a punto per lunga esperienza le regole più idonee in rapporto al migliore materiale disponibile (verifica dei comportamenti delle opere nei tempi lunghi onde eliminare le soluzioni meno durevoli), ogni maestro sapeva esattamente come sbozzare o semplicemente spaccare o anche soltanto scegliere dalla fornitura di cava gli elementi adatti in ogni posizione. La complessità non era quindi nel procedimento, ma in quella abilità di giudicare velocemente il meglio senza interruzioni: programmi mentali usati in questo modo esistono, d'altra parte, anche in altri settori delle costruzioni, come nel caso della produzione dei mosaici. Certo è che è assai più difficile riscoprire le regole, ma soprattutto le diverse attenzioni pratiche, di una tecnica complessa rispetto a quelle dell'opera quadrata, una volta morto senza apprendisti l'ultimo maestro.

Non si possono classificare come opere murarie i paramenti litici esterni che non presentano un corrispondente paramento interno, ma che creano una ampia struttura assieme ad un terrapieno, come si vede nella torre della prima fase urbana di Gerico (VII millennio), nelle mastabe egiziane $\mathrm{o}$, in Occidente, nel santuario di Monte D'Accoddi (IV millennio), costruito al modo delle ziqqurat mesopotamiche. L'apparente muro non ha, infatti, vere funzioni statiche in questi casi, ma piuttosto quella di rendere quasi verticale e di proteggere il fianco del riempimento dagli agenti atmosferici. Queste costruzioni hanno anche rappresentato, però, i primi tentativi di grandi opere architettoniche attraverso le quali si è molto probabilmente imparato a costruire i muri veri e propri.

Questa evoluzione è praticamente avvenuta, forse per la prima volta, in Egitto, come si può vedere nel complesso regale di Saqqara (III millennio). Quello che è più interessante è che nelle prime architetture egizie sono presenti già tutte le soluzioni tecniche alle quali si rifanno i numerosi tipi murari posteriori, se si esclude forse l'opera poligonale a elementi con più di quattro angoli fatto che potrebbe essere dipeso dalla natura petrografica de materiali disponibili.

Sembra, cioè, che in Egitto siano state sperimentate subito buona parte delle diverse tecniche che sono state per millenni, fino ai giorni nostri, alla base della complessa tipologia dei muri in tutti i continenti; come se, una volta capito quali caratteristiche statiche (regole dell'arte) dovesse avere un muro, si sia cercato in quanti modi fosse possibile raggiungerle, agendo da un lato sulla scelta e preparazione, se occorre anche sofisticata a fini estetici e simbolici, degli elementi litici, e dall'altro lato sulla loro messa in opera e sulle evidenti influenze reciproche fra i due aspetti. Le fonti antiche riportano d'altra parte quale peso hanno avuto le tecniche di estrazione, lavorazione e costruzione litica degli Egiziani sulle civiltà dell'area mediterranea.

\section{CRITERI PER UNA BUONA CLASSIFICAZIONE DEI MURI}

Se si tiene conto delle possibili risorse naturali, di come esse siano state estratte, lavorate e messe in opera per una buona funzione statica, oltre che estetica, è utile e necessario non partire dalla descrizione oggettiva dell'opera finita, ma da quella di ogni sua parte tecnicamente importante, indipendentemente l'una dall'altra: gli elementi costruttivi (A), la loro disposizione (B), i giunti fra elemento ed elemento $(\mathrm{C})$, la terza dimensione $(\mathrm{D})$.

A1. La natura petrografica degli elementi litici è utile perché determina in buona parte le forme grezze (facce parallele degli stratiformi e degli scistosi), la tecnica di lavorazione più facile e meno costosa (durezza, tenacità, sfaldabilità), la messa in opera più durevole (orientazione dei «versi»), oltre ai valori estetici (colore, effetti di superficie, espressione di robustezza).

A2. La forma geometrica degli elementi impiegati può essere classificata in sei categorie: a) quadrangolare (con angoli almeno in parte non retti, ma con due facce parallele fra loro); b) squadrata (con tutti gli angoli retti); c) squadrata a $L$ (come l'altra, ma con uno o più lati che presentano una risega ortogonale); d) poligonale (con più di quattro angoli); e) tondeggiante (tendenti allo sferico, all'ellittico o al multiradiale); f) a scaglie (esistono in qualche caso paramenti eseguiti con scaglie di pietra da raccolta o provenienti da altre lavorazioni).

A3. La forma lavorata, determinata cioè dalle lavorazioni preparatorie a cui gli elementi sono stati sottoposti, è variabile: a) pietrame raccolto (spietratura dei campi e dei pascoli); b) pietrame di cava (tracce di piccone o dei cunei); c) elementi a spacco (eseguito lungo un "verso» o un "pelo" con la mazzetta usata di spigolo che lascia un piccolo cratere di roccia macinata); d) bozze (elementi sbozzati con martello, o punta grossa e mazzuolo, che lasciano scheggiature tipiche con bulbi negativi di percussione o con foro inclinato); e) conci (elementi con spigoli vivi rettilinei, ottenuti a scalpello inclinato e martello e con facce di contatto perfettamente piane); f) ciottoli naturali; g) ciottoli spaccati; h) ciottoli sbozzati.

È evidente che possono esistere conci quadrangolari, conci squadrati ad L e conci poligonali. Le bozze possono 
essere solo quadrangolari o poligonali. Di forma quadrangolare possono essere anche elementi di pietrame a spacco di cava o da raccolta, se si tratta di rocce stratificate, mentre le rocce di origine magmatica forniscono più facilmente, con le stesse lavorazioni, forme poligonali.

B. Le disposizioni degli elementi litici nella tessitura muraria viste sulle superfici esterne sono raggruppabili in due categorie principali: muri a corsi e quelli senza corsi. Per corso si intende quando, una pietra a fianco all'altra, la sequenza orizzontale può essere seguita da un capo all'altro dell'unità stratigrafica muraria, a prescindere dalla natura e dalla forma geometrica e lavorata degli elementi impiegati, e qualsiasi variazione di organizzazione possa avvenire all'interno del corso stesso.

B1. I corsi è più facili classificarli a partire da quelli formalmente perfetti, perché più semplici da descrivere e capire, anche se più costosi da realizzare: a) regolari (con altezza costante degli elementi da un capo all'altro del muro); b) alternati (conci grandi, ma di riolotto spessore, dispoti alternativamente in piano e in verticales dentro corsi regolari); c) sdoppiati (quando l'altezza del corso viene raggiunta in alcuni punti con uno o più elementi sovrapposti); d) con scalino (dopo elementi alti tutti uguali si passa ad elementi tutti uguali tra loro, ma di altezza differente dai precedenti: l'elemento a contatto dello scalino nel corso successivo deve essere sagomato ad L); e) ad altezza non costante, o budinate (le non eccessive differenze di altezza degli elementi creano contorni oscillanti nel corso, compensati nello strato successivo); f) ripianati ogni $x$ strati (le diverse altezze degli elementi vengono compensate in due o più starti discontinui, che non costituiscono cioè dei corsi, per ottenere un piano regolare); g) reticolati (con elementi quadrangolari uguali tra loro appoggiati di spigolo, per rotazione di $45^{\circ}$ del piano di posa); h) a spina di pesce (elementi a scaglia o ellittici di piccole dimensioni, che solo in questo modo possono creare un paramento sufficientemente legato).

È chiaro che i corsi regolari e sdoppiati possono essere realizzati con elementi squadrati o sbozzati e a spacco di forma quadrangolare, ma anche con pietrame di cava, quando è in strati naturali molto regolari e di altezza opportuna; i corsi a scalino, invece, solo con i primi due tipi di lavorazione. I corsi budinati sono in prevalenza ottenuti con elementi a forma tondeggiante (grossi ciottoli naturali o spaccati), ma anche con forme quadrangolari ad altezze un po' diverse. Certi corsi budinati hanno una forte parentela con l'opera poligonale. Nei corsi ripianati possono essere impiegati assieme elementi di ogni forma geometrica: ogni corso è come una fascia di tecnica complessa.

Delle tecniche a corsi è necessario descrivere anche se essi sono uguali o differenti per altezza nella stessa unità strati- grafica muraria, e se le differenti altezze si susseguono casualmente o con qualche regola (per esempio dalla più grande alla più piccola, per poi riprendere la sequenza): sono informazioni importanti per conoscere l'organizzazione del cantiere.

A questo proposito può essere utile tenere presente la notevole differenza di organizzazione che richiedono le lavorazioni e messe in opera di conci (spigoli rettilinei, cioè e facce piane) con forme squadrate, squadrate ad $\mathrm{L}$ e poligonali. Nel primo caso, infatti, lo scalpellino può preparare, come si è già detto, l'intero muro a terra per essere in seguito montato. Negli altri due casi ogni concio deve venire preparato dallo scalpellino mano a mano che la messa in opera procede, perché è necessario fare una sagoma (dima o sesta) degli angoli, per i poligonali, e del profilo ben preciso dello scalino, per i conci ad L.

B2. Per i muri senza corsi, in attesa che venga messa a punto un programma informatico per la loro analisi del genere «reti neurali», si può cercare di estrarre, dalla notevole quantità di varianti esistente quelle con qualche evidenza riconducibile a delle geometrie lineari. Gli esperimenti fatti finora con i frattali e con analisi matematiche, hanno dimostrato che in nessun caso le tecniche murarie sono casuali, ma non è ancora possibile ricavare con questi strumenti dei parametri per una classificazione.

La percezione umana può vedere differenze in quasi tutte le opere murarie complesse, ma non riesce ad organizzarle in modo ripetibile, tranne per alcune tessiture: a) opera poligonale (eseguita con elementi grandi di forma poligonale bene incastrati fra di loro, con il possibile uso di elementi piccoli con la funzione di zeppe); b) a pilastri (alcuni elementi più grandi sono ogni tanto sovrapposti a creare delle nervature verticali con immorsature laterali); c) ad alveare (gli elementi più grandi sono a contatto tra loro verso gli spigoli creando celle riempite con elementi più piccoli). Nelle tessiture con distribuzione omogenea è possibile fornire: d) diagramma granulometrico (i rapporti quantitativi fra le varie classi dimensionali degli elementi); e) andamento dei giunti orizzontali (deviazioni standard della curva che collega tra loro tutti i giunti orizzontali prossimali presenti lungo uno stesso livello costruttivo)

C. I giunti tra elemento ed elemento costituiscono, come si è detto, assieme alla forma e lavorazione degli elementi stessi ed alla loro disposizione nell'apparecchio murario, la terza caratteristica tecnicamente importante per tutti i tipi osservati sulle superfici esterne. Il dato più interessante è l'estensione dei contatti fra pietra e pietra: essa è però abbastanza ben visibile nei muri a secco, $o$ in quelli a calce con conci, in quanto le facce spianate consentono giunti molto sottili, e gli spigoli vivi evitano che la malta ricopra le smussature, facendo apparire più larghi i giunti. 
Tenuto conto di questi problemi di osservazione, i dati rilevabili sono: a) lo spessore (in millimetri con il minimo ed il massimo per ogni giunto); b) la consistenza della malta (quanto aderisce e quanto resiste allo schiacciamento); c) le zeppe orizzontali (percentuale di giunti che ne hanno uno, due o più); d) le zeppe verticali (percentuale); e) le zeppe di nodo trilitico (percentuale; sono prevalenti e importanti nelle opere poligonali realizzate senza conci).

D. Come si è detto in partenza, non si può parlare veramente di tecnica muraria senza conoscere anche la terza dimensione di una struttura la cui tessitura bidimensionale è visibile sulla superficie esterna. Il dato più importante dal punto di vista statico è costituito dal sapere se tale tessitura corrisponda ad un paramento costruito in modo diverso dalla parte interna del muro, o faccia parte di una tecnica uguale su tutto lo spessore. In questo secondo caso sarebbe più semplice valutare il comportamento dell'intera struttura anche dalle superfici esterne.

Le osservazioni possibili sono: a) spessori delle pietre nei cantoni (quando questi siano costruiti con la stessa tecnica del muro); b) spessore totale del muro (per vedere se, sottratti gli spessori delle due tessiture visibili, esista uno spazio sufficiente per una tecnica ignota).

L'esperienza sui muri visibili anche all'interno insegna comunque che: i muri che sono opera completa del muratore, e quelli complessi in modo particolare, sono realizzati su tutto lo spessore con la stessa tecnica; quelli preparati dallo scalpellino no, a meno che i due paramenti occupino tutto lo spessore. Non sono mai costruiti con la stessa tecnica i muri con paramenti molto sottili, come i corsi a spina di pesce in piccoli ciottoli. Non è detto però che in questi casi l'interno sia sempre realizzato a sacco con un calcestruzzo, creando un diverso comportamento statico e dinamico; anzi, nella maggior parte dei casi verificati, l'interno dei paramenti è stato costruito a strati costipati di pietre, non usate negli esterni, e calce.

Questo lavoro, che tratta in modo nuovo gli stessi manufatti già discussi in diversi lavori precedenti, non comporta che quelli già pubblicati siano superati; significa soltanto che nel tempo è stato progressivamente cambiato il punto di osservazione di uno stesso scenario complesso, ed ogni punto fornisce le sue interpretazioni.

\section{Riferimenti bibliografici}

MANNONi T., 1976, L'analisi delle tecniche murarie medievali, in Atti del Convegno Internazionale di Archeologia Medievale (Erice 1974), Palermo, pp. 291-300.

PARENTI R., 1988, Sulle possibilità di datazione e di classificazione delle murature, in Archeologia e Restauro dei Monumenti, a cura di R. Francovich e R. Parenti, Firenze, pp. 280-304.

Cagnana A., Mannoni T., 1995, Materiali e tecniche nelle strutture murarie di Luni. Risultati preliminari, in Quaderni del Centro Studi Lunensi, 1 n. s., pp. 137-164.

MANNONI T., 1997, Il problema complesso delle murature storiche in pietra. 1 Culturu materiale e cronotipologia, in Archeologia dell'Architettura II, pp. 15-24.

Mannoni T., Cicirello C., 1998, Atlante dei tipi costruttivi murari dell'ltalia Settentrionale, in Atti del seminario. La protezione del patrimonio culturale. La questione sismica (Roma 1995), Roma, pp. 87-99.

MANNONI T., 1998, Il problema complesso delle murature storiche. Regole costruttive e resistenze meccaniche, in Atti del seminario. Lo spessore storico in architettura tra conservazione, restauro e distruzione (Milano 1995), Milano, pp. 59-64. 